\title{
Hybrid Plasmonic and Pyroelectric Harvesting of Light Fluctuations
}

Mina Shiran Chaharsoughi, Daniel Tordera, Andrea Grimoldi, Isak Engquist, Magnus Berggren, Simone Fabiano and Magnus Jonsson

The self-archived postprint version of this journal article is available at Linköping University Institutional Repository (DiVA):

http://urn.kb.se/resolve?urn=urn:nbn:se:liu:diva-148574

N.B.: When citing this work, cite the original publication.

This is a copy of the published version of the following article:

Chaharsoughi, M. S., Tordera, D., Grimoldi, A., Engquist, I., Berggren, M., Fabiano, S., Jonsson, M., (2018), Hybrid Plasmonic and Pyroelectric Harvesting of Light Fluctuations, Advanced Optical Materials. https://doi.org/10.1002/adom.201701051

which has been published in final form at:

Original publication available at:

https://doi.org/10.1002/adom.201701051

Copyright: Wiley-Blackwell

http://wiley.com/ 
DOI: 10.1002/adom.201701051

Article type: Communication

\section{Hybrid Plasmonic and Pyroelectric Harvesting of Light Fluctuations}

Mina Shiran Chaharsoughi, Daniel Tordera ${ }^{\dagger}$, Andrea Grimoldi, Isak Engquist, Magnus Berggren, Simone Fabiano, Magnus P. Jonsson*

M. Shiran Chaharsoughi, D. Tordera, A. Grimoldi, I. Engquist, M. Berggren, S. Fabiano, M. P. Jonsson

Laboratory of Organic Electronics, Department of Science and Technology, Linköping University, Norrköping, 60221, Sweden

Email: magnus.jonsson@liu.se

†Present address: Holst Centre/TNO, High Tech Campus 31, 5605 KN Eindhoven, The Netherlands

Keywords: Plasmonic heating, gold nanodisks, pyroelectric copolymer, solar energy harvesting

State-of-the-art solar energy harvesting systems based on photovoltaic technology require constant illumination for optimal operation. However, weather conditions and solar illumination tend to fluctuate. Here, a device is presented that extracts electrical energy from such light fluctuations. The concept combines light-induced heating of gold nanodisks (acting as plasmonic optical nanoantennas), and an organic pyroelectric copolymer film (poly(vinylidenefluoride-co-trifluoroethylene), $\mathrm{P}(\mathrm{VDF}-\mathrm{TrFE})$ ) that converts temperature changes into electrical signals. This hybrid device can repeatedly generate current pulses, not only upon the onset of illumination $\left(13 \mu \mathrm{A} \mathrm{m}^{-2}\right.$ by sunlight), but also when illumination is blocked. Detailed characterization highlights the key role of the polarization state of the copolymer, while the copolymer thickness has minor influence on performance. The results are fully consistent with plasmon-assisted pyroelectric effects, as corroborated by combined optical and thermal simulations that match the experimental results. Owing to the tunability of 


\section{WILEY-VCH}

plasmonic resonances, the presented concept is compatible with harvesting near infrared light while concurrently maintaining visible transparency.

Solar irradiance at the ground typically fluctuates due to natural processes such as clouds moving over the sky or tree branches swinging in the wind. While this affects plants and cause variations in their photosynthetic activity, ${ }^{[1]}$ such light fluctuations may also be utilized and harvested through light-induced thermal energy conversion. ${ }^{[2]}$ Thermal energy can be converted directly to electricity through thermoelectric or pyroelectric energy harvesting. ${ }^{[3]}$ While thermoelectric systems transform a temperature gradient to electrical energy, pyroelectric materials instead enable conversion of temporal variations in temperature to electricity. ${ }^{[2-3]}$ Interestingly, many natural biomaterials possess pyroelectric properties, including the leaves of the palm-like plant Encephalartos. ${ }^{[4]}$ While the physiological implications of pyroelectricity in plants and other biomaterials are still poorly understood, ${ }^{[4-5]}$ the effect can already be utilized in artificial devices for applications including light detection $^{[2 \mathrm{~d}, 6]}$ and energy harvesting. ${ }^{[3,7]}$

Pyroelectric polymers have attracted considerable attention owing to their mechanical flexibility, easy processing, and low cost. ${ }^{[4-5]}$ While there are a number of pyroelectric polymers, such as poly(vinylchloride) (PVC) and Nylon 11, poly(vinylidene fluoride) (PVDF) and its copolymer poly(vinylidenefluoride-co-trifluoroethylene) $((\mathrm{P}(\mathrm{VDF}-\mathrm{TrFE}))$ are particularly promising due to high pyroelectric coefficients and good chemical stability. ${ }^{[2 \mathrm{a}, 2 \mathrm{c} \text {, }}$ ${ }^{2 \mathrm{~d}, 8]}$ Typical application areas for these polymers include heat sensing, thermal imaging, and fire alarms. ${ }^{[2 \mathrm{a}]}$ In addition, these polymers have been significantly explored based on their piezoelectric properties to harvest mechanical energy. ${ }^{[3,9]}$ Their combination with plasmonic nanostructures was only recently reported, for laser-based patterned phase-control of 


\section{WILEY-VCH}

ferroelectric polymers. ${ }^{[10]}$ However, plasmonic heating was, to our knowledge, not previously investigated for pyroelectric energy harvesting.

Here, we present a concept that converts temporal fluctuations in sunlight to electrical energy. The device combines a plasmonic metasurface consisting of gold nanodisks with a thin organic $\mathrm{P}(\mathrm{VDF}-\mathrm{TrFE})$ copolymer film. The plasmonic nanostructures strongly absorb light through resonant excitation of plasmons (collective charge oscillations), which leads to local heating of both the nanostructure and the surrounding environment. ${ }^{[11]}$ Such light-induced plasmonic heating can enable a wide range of applications, including solar-powered autoclaving, ${ }^{[12]}$ plasmon-driven thermophoresis, ${ }^{[13]}$ seawater catalysis, ${ }^{[14]}$ plasmonicthermoelectric light detection ${ }^{[15]}$, and desalination concepts. ${ }^{[14]}$ In our hybrid plasmonicpyroelectric device (referred to as hybrid device below), plasmonic heating of the gold nanodisk array modulates the temperature of the pyroelectric polymer. The polymer then converts these temperature-changes to electrical signals through the pyroelectric effect.

We demonstrate the concept by designing a device that powers an external load connected to the hybrid device, and by characterizing its performance in detail. Combined optical and thermal simulations of the hybrid device are in agreement with the experimental results and corroborate the proposed working mechanisms.

Figure 1a schematically shows the hybrid device architecture. The device comprises an array of $160 \mathrm{~nm}$ in diameter and $30 \mathrm{~nm}$ thick gold nanodisks (with $3 \mathrm{~nm}$ Ti as adhesion layer) deposited on a patterned indium tin oxide (ITO) electrode on a glass substrate. On top of the nanodisk surface there is a $660 \mathrm{~nm}$ thick layer of $\mathrm{P}(\mathrm{VDF}-\mathrm{TrFE})$ copolymer topped with a 30$\mathrm{nm}$ thick gold film acting as top electrode. Figure $1 \mathrm{~b}$ shows a top view photograph of a device. The red dashed line indicates the active area $\left(3 \times 3 \mathrm{~mm}^{2}\right)$, which is the region where the sandwiched bottom and top electrode overlap. We characterized the samples by scanning electron microscopy (SEM) and extinction spectroscopy. Figure 1c shows SEM images of a 


\section{WILEY-VCH}

typical sample after fabrication of gold nanodisks. The disks are around $160 \mathrm{~nm}$ in diameter and distributed in a short-range order with a characteristic spacing of $325 \mathrm{~nm}$ between neighboring nanodisks. The latter was determined from the peak in the radial distribution function of the SEM images (see Figure S1c). The short-range order originates from the nanofabrication process based on hole-mask colloidal lithography. This method enables rapid production of large nanostructured plasmonic nanodisk surfaces ${ }^{[16]}$ with optical properties similar to those of typical periodic nanodisk arrays (as used in the simulations below to resemble the experimental surfaces). ${ }^{[17]}$ Figure $1 \mathrm{~d}$ presents the plasmonic response before and after addition of the $\mathrm{P}(\mathrm{VDF}-\mathrm{TrFE})$ copolymer layer. The bare nanodisk sample shows a strong plasmonic extinction peak at $731 \mathrm{~nm}$ (black line), which is red-shifted to $804 \mathrm{~nm}$ after depositing the P(VDF-TrFE) layer (blue line). The red-shift is due to the increase in the refractive index of the surrounding medium of the nanodisks from 1 (air) to about 1.42 $[\mathrm{P}(\mathrm{VDF}-\mathrm{TrFE})] \cdot{ }^{[18]}$ In turn, this makes the structure largely transparent in the visible, while strongly interacting with light in the near infrared tail of the solar spectrum, making the concept suitable also for transparent applications. While we employ these nanodisk metasurfaces to demonstrate the concept, other types of plasmonic surfaces may be explored in future work.

Simulated extinction spectra obtained by the finite-difference time-domain (FDTD) method (dashed black and blue lines, see SI for details) agree well with the experimental results, showing resonance peaks at $750 \mathrm{~nm}$ and $815 \mathrm{~nm}$ for gold nanodisks without and with P(VDFTrFE) layer, respectively. Experimental extinction spectra of a sample without nanodisks but with the $\mathrm{P}(\mathrm{VDF}-\mathrm{TrFE})$ layer (red line) verifies that the copolymer is transparent in the whole wavelength region and it will contribute negligibly to the total absorption of the device.

The hybrid plasmonic pyroelectric concept requires polarized $\mathrm{P}(\mathrm{VDF}-\mathrm{TrFE})$ layers with a residual electric-dipole moment. Without a net dipole moment, the film would not provide 


\section{WILEY-VCH}

any effective pyroelectric properties. ${ }^{[2 b]}$ However, after the fabrication process, the P(VDFTrFE) layer contains randomly oriented dipoles, which results in zero residual electric-dipole moment over the film. ${ }^{[2 \mathrm{~b}]}$ While the direction and magnitude of the spontaneous polarization is fixed after fabrication for most materials, P(VDF-TrFE) allows for in situ alignment of the domains by applying over the film an electric field stronger than the coercive field. ${ }^{[2 b, 19]}$

This so-called poling process is schematically illustrated in Figure 2a for the P(VDF-TrFE) layer in the hybrid device. The poling process aligns the dipoles with the applied electric field and results in a corresponding residual spontaneous polarization $\left(P_{\mathrm{s}}\right)$ in the copolymer layer. Figure $2 \mathrm{c}$ shows the experimental poling behavior for a hybrid device (660-nm thick P(VDFTrFE) layer, black line) and a control device with the same structure, but lacking the gold nanodisk array (blue line). Small arrows on the graph indicate the direction of the voltage sweep. Scanning the voltage from $0 \mathrm{~V}$ to positive voltages leads to a transient current peak around $46 \mathrm{~V}$ (electric field around $70 \mathrm{~V} \mu \mathrm{m}^{-1}$ over the 660-nm thick film), which corresponds to the alignment of the domains and the formation of a net polarization over the film. Scanning the voltage to negative potentials flips the polarization, again resulting in a transient current (with a peak around $-33 \mathrm{~V}$ ). The polarization cycle was repeated several times to maximize the film quality and polarization. Importantly, no transient current peaks were observed when reducing the voltage back to $0 \mathrm{~V}$, which shows that the alignment and polarization remained also after removing the bias voltage. The results for the hybrid device and the control device are very similar. The poling was triggered at the same voltage (for the same copolymer thickness, here $660 \mathrm{~nm}$ ), indicating that the nanodisks do not affect the coercive field or interfere with the process. Although Figure 2c shows higher current for the hybrid device than for the control device, this varied from device to device with no clear trends. Interestingly, both systems show asymmetries in the current loops, which are attributed to asymmetries in the copolymer layer and/or the device architecture. ${ }^{[20]}$ 


\section{WILEY-VCH}

Importantly, the results confirm successful dipole alignment after poling for both systems.

To investigate pyroelectric effects upon intermittent illumination, we measured the shortcircuit current density and corresponding accumulated charge density (absolute values) for a hybrid device before and after polarization, and for a control device without plasmonic structures (Figure $2 \mathrm{~b}$ and $2 \mathrm{~d}$ ). As expected, the non-polarized device (green line) shows almost no response upon illumination with simulated sunlight (1 Sun, $1000 \mathrm{~W} \mathrm{~m}^{-2}$ ). Only a tiny positive current and accumulated charge density can be observed during illumination. The results after polarization are highly different (black line). In this case opening the shutter to the light source gives a rapid increase in current density $\left(13 \mu \mathrm{A} \mathrm{m}^{-2}\right)$ followed by a slow decrease towards zero. Turning off the illumination leads to a very similar response, but with reversed current density direction. The polarized control device shows similar behavior, but with lower current density peak of about $6.7 \mu \mathrm{A} \mathrm{m}^{-2}$ (blue line) leading to accumulating 377 $\mu \mathrm{C} \mathrm{m}^{-2}$ charge per area at positive electrode. The signals for the control device are attributed primarily to heating of the thin top gold electrode. The results are in agreement with expectations based on the pyroelectric effect, for which the short-circuit current density $\left(\frac{i_{p}}{A}\right)$ is given by: $[2 \mathrm{a}, 2 \mathrm{~b}]$

$$
\frac{i_{p}}{A}=\frac{1}{A}\left(\frac{\mathrm{d} Q}{\mathrm{~d} t}\right)=P\left(\frac{\mathrm{d} T}{\mathrm{~d} t}\right)
$$

Here, $Q$ is the charge, $P$ is the pyroelectric coefficient, $A$ is the effective area, and $\mathrm{d} T / \mathrm{d} t$ is the rate of temperature change. Hence, the observed positive current density pulse is a result of rapid temperature increase $(\mathrm{d} T / \mathrm{d} t>0)$ upon illumination, followed by a monotonic decrease as the system reaches its equilibrium temperature $(\mathrm{d} T / \mathrm{d} t=0)$. The mechanism can be understood from the temperature dependence of that spontaneous polarization of $\mathrm{P}(\mathrm{VDF}-\mathrm{TrFE})$. An increase in temperature reduces the spontaneous polarization, as the dipoles in the material lose their equilibrium positions due to increased thermal vibration. ${ }^{[2 a, 19 a]}$ In turn, reducing the 


\section{WILEY-VCH}

spontaneous polarization leads to a reduction in charge density on the electrodes, which induces a compensating transient electric current between the two electrodes, corresponding to a total accumulated transferred charge of $646 \mu \mathrm{C} \mathrm{m}^{-2}$. Likewise, switching off the light stops the plasmonic heating, resulting in a decrease in temperature $(\mathrm{d} T / \mathrm{d} t<0)$ and a corresponding negative current density pulse (about $-13 \mu \mathrm{A} \mathrm{m}^{-2}$ ). This lead to an increase in the accumulated charge density on the electrodes of $558 \mu \mathrm{C} \mathrm{m}^{-2}$. This reversed current pulse upon blocking the light is then related to the spontaneous polarization regaining its initial equilibrium value at room temperature. The somewhat larger measured transferred charge density for the light on pulse is attributed to a small non-zero current that maintained also at long times upon illumination, and may be related to thermoelectric effects. ${ }^{[21]}$

The control device shows a lower negative current density pulse (about $-9 \mu \mathrm{A} \mathrm{m}^{-2}$ ) and lower amount of accumulated charge density $\left(270 \mu \mathrm{C} \mathrm{m}^{-2}\right)$.

For comparison with the experimental results, we employed combined optical and heat transfer simulations to model the plasmon-induced pyroelectric short-circuit current density and the accumulated absolute change in charge density. As explained in detail in the Supporting Information, the FDTD model was first used to predict the absorbed power of the hybrid device upon solar illumination. The resulting absorbed powers then acted as heat sources in heat transfer simulations based on the finite element method (FEM). We performed time-dependent simulations for hybrid devices using the same geometry as the experimental devices (including both nanodisks and top gold electrode) and where the heat source (i.e. the light source) was turned on and off as in the experiments. Figure S3a shows the resulting temperature evolution and Figure S3b presents the corresponding rate change in temperature (dT/d $t$ ). As shown, the kinetics of $\mathrm{d} T / \mathrm{d} t$ resemble the experimental short-circuit current density results (Figure 2b). The plasmon-induced pyroelectric current density response can now be calculated using Equation 1, using $P=55 \times 10^{-6} \mathrm{C} \mathrm{m}^{-2} \mathrm{~K}^{-1}$ for $\mathrm{P}(\mathrm{VDF}-\mathrm{TrFE})^{[6]}$ and an 


\section{WILEY-VCH}

effective area $A=9 \times 10^{-6} \mathrm{~m}^{2}$. The resulting calculated pyroelectric current density and accumulated charge density are plotted as the red dashed lines in Figure $2 \mathrm{~b}$ and $2 \mathrm{~d}$, respectively. There is good match between the simulated and experimental results, not only in absolute current values and accumulated charge, but also in the kinetics of the evolution in current and the accumulated charge density.

Next, we demonstrate energy harvesting of hybrid devices upon simulated fluctuating solar illumination. The devices were connected to an external $9 \mathrm{M} \Omega$ resistor, and the voltage was measured across this load over time. Figure 3a shows the corresponding output voltage across the load for a hybrid device (black line) and for a control device (blue line) under light on/off states. The response for the non-polarized hybrid device is presented as the green line and as expected shows very small signals upon changes in illumination. By contrast, the voltage across the load for the polarized hybrid device reached $1.5 \mathrm{mV}$ after switching on the light source and $-1.4 \mathrm{mV}$ when switching it off. The pulse shape resembles that of the short-circuit current and the results are in agreement with plasmon-powered pyroelectrics. The behavior and pulse shape for the control device without plasmonic nanodisks was similar as for the hybrid device, but with lower signals and output voltages.

Figure $3 \mathrm{c}$ presents the corresponding power densities $(\mathrm{Pd})$ provided by the different devices. The maximum power density of the polarized hybrid device was $0.027 \mu \mathrm{W} \mathrm{m}^{-2}$ and $0.024 \mu \mathrm{W}$ $\mathrm{m}^{-2}$ under light switching on and off, respectively. The control device could only generate about one sixth and one third of the peak power density generated by the hybrid device under the same conditions. This highlights the important role of the plasmonic metasurface to induce efficient light-to-heat conversion for the hybrid device, resulting in rapid changes in temperature (dT/dt) upon illumination (Figure S3b), and correspondingly higher pyroelectric currents and energy harvesting performance. As expected from the low voltages presented in Figure 3a, the power density generated by the non-polarized hybrid device was almost zero. Hence, while the plasmonic array provided effective heating and corresponding high $\mathrm{d} T / \mathrm{d} t$ 


\section{WILEY-VCH}

also for this device, there was no observable pyroelectric response because of the negligible residual electric dipole moment in the $\mathrm{P}(\mathrm{VDF}-\mathrm{TrFE})$ layer.

According to equation (1) the thickness of the pyroelectric film should not affect the generated current. Figure $3 \mathrm{~b}$ shows the output voltage generated by hybrid devices with $\mathrm{P}(\mathrm{VDF}-\mathrm{TrFE})$ of different thicknesses. The results are indeed very similar for all thicknesses, and the fact that there is no decrease in performance even for the lowest thickness of $165 \mathrm{~nm}$ is promising for enabling ultrathin hybrid energy harvesting devices. In this respect, we note that the thickness does not affect the spontaneous polarization or the pyroelectric coefficient of the polymer film, ${ }^{[2 b]}$ although small differences can occur due to differences in secondary and tertiary pyroelectric effects related to strain and shear stress induced by thermal expansion and non-uniform heating in the copolymer layer. ${ }^{[2 a]}$ A more systematic investigation shows a small variation in the mean value of the integrated pyroelectric power density generated for hybrid devices with different thicknesses upon illumination (Figure 3d, black dots and line). This variation is attributed to small differences in the amount of solar light absorbed by devices of different thicknesses. Indeed, the experimental trend matches the trend predicted based on differences in the simulated absorbed solar power density for different thicknesses (red dots and dashed line). While these differences in total solar absorption are small, a closer inspection shows that the polymer thickness can be used to control the spectral response, as attributed to differences in optical cavity effects from reflections from the top gold electrode (see Figure S2).

Finally, we demonstrate energy harvesting by the hybrid device in conditions representing natural light fluctuations. For this, a leaf of a plant acted as semitransparent shutter to block light from a solar simulator in controlled and random ways. In the controlled approach, the leaf blocked the light in on/off time spans of $30 \mathrm{~s}$ (controlled manually). In the random approach, the leaf was swinging in the air turbulence caused by a fan (Figure 4a), resulting in 


\section{WILEY-VCH}

more rapid and random light intensity fluctuations. Similar to the previous measurements, we monitored the output voltage across a $9 \mathrm{M} \Omega$ resistor connected to the device. Figure $4 \mathrm{~b}$ and $4 \mathrm{~d}$ show the output voltage and power density upon controlled light fluctuations by the leaf for a polarized hybrid device (black line) and for a non-polarized hybrid device (green line). In agreement with the results above, the polarized hybrid device produced positive and negative output voltage pulses and corresponding generated power densities upon light and shade, respectively. By contrast, the non-polarized hybrid device showed negligible signals. Figure $4 \mathrm{c}$ and $4 \mathrm{e}$ instead shows the response upon natural random light fluctuations from the leaf swinging in the wind. Again, the polarized hybrid device (black line) produced clear output voltage and power density signals while the signals generated by the non-polarized device were almost zero. These results show that the polarized hybrid device can generate output voltage in surroundings where the light is fluctuating through natural processes such as swinging plant leaves and moving clouds.

In summary, we have presented a hybrid device that harvests energy from fluctuations in sunlight, based on plasmon-induced pyroelectric effects. The concept takes advantage of the strong light absorption of plasmonic optical nanoantennas to modulate the temperature of a pyroelectric $\mathrm{P}(\mathrm{VDF}-\mathrm{TrFE})$ layer, enabling extraction of energy through an external load under fluctuating solar irradiation. The results are in agreement with combined optical and heat transfer simulations. The concept provides a means to extract energy from changes in illumination conditions, which makes it interesting as complement to other harvesting systems based on continuous irradiation, such as traditional photovoltaic solar cells or thermoelectric devices. Future devices may also benefit from implementing rectifying circuits and capacitors or supercapacitors for storage of the generated energy for use upon demand. ${ }^{[2 \mathrm{a}]}$ Combined with large tunability of plasmonic resonances throughout the visible and the infrared 


\section{WILEY-VCH}

wavelength regimes, the concept may also find use in wireless sensors for detection of electromagnetic radiation and other applications.

\section{Experimental Section}

Hybrid device fabrication: The hybrid devices were fabricated from glass substrates containing a 90-nm thick patterned ITO bottom electrode. To clean the substrates, they were first ultrasonicated in soap water for $5 \mathrm{~min}$ and two times in de-ionized water for 5 min each. Next, they were rinsed with isopropanol and dried with a $\mathrm{N}_{2}$ stream. This was followed by UV-ozone treatment for 15 min to remove organic contaminations. After cleaning, an array of plasmonic gold nanodisks (diameter of $160 \mathrm{~nm}$ and thickness of $30 \mathrm{~nm}$, and with $3 \mathrm{~nm}$ titanium as adhesion layer) were produced by hole-mask colloidal lithography. This fabrication method has been described in detail previously ${ }^{[16]}$ and provides large-area shortrange ordered nanodisk arrays based on a bottom-up self-assembly process. Next, the nanodisk array was embedded in a P(VDF-TrFE) layer by spin coating of $4 \mathrm{wt} . \% \mathrm{P}(\mathrm{VDF}-$ TrFE) copolymer solution (P(VDF-TrFE) (70:30 mol\%) pellets in diethyl carbonate) at 4000 rpm for $30 \mathrm{~s}$. To determine the thickness of the copolymer layer, the step height over the edge of a scratch was measured using Dektak 3ST profilometer. The device was then annealed at $130{ }^{\circ} \mathrm{C}$ for 10 min to improve the crystallinity of the P(VDF-TrFE) layer. Finally, a 30-nm thick gold top electrode was shadow-evaporated. In order to investigate copolymer thickness effects, devices with different $\mathrm{P}(\mathrm{VDF}-\mathrm{TrFE})$ thicknesses were also prepared. By repeating a series of spin coating and annealing steps, eight hybrid devices were fabricated for four $\mathrm{P}(\mathrm{VDF}-\mathrm{TrFE})$ thicknesses $(165 \mathrm{~nm}, 320 \mathrm{~nm}, 470 \mathrm{~nm}, 660 \mathrm{~nm})$.

Poling process: After the annealing process, the $\mathrm{P}(\mathrm{VDF}-\mathrm{TrFE})$ is in the polar $\beta$-phase, characterized by randomly oriented domains ${ }^{[8 a]}$ but they can be aligned by applying an electric field of $100 \mathrm{~V} \mathrm{\mu m}^{-1}$ or more over the film ${ }^{[8]}$. This so-called poling process was 


\section{WILEY-VCH}

performed using cyclic voltammetry. An electric field of $\pm 100 \mathrm{~V} \mathrm{\mu m}^{-1}$ with the $3 \mathrm{~V} \mathrm{~s}^{-1}$ was applied to the devices in a number of cycles using a Keithley 4200-SCS parameter analyzer.

Optical and morphological characterization: The UV/Vis/NIR spectroscopy (PerkinElmer Instruments Lambda 900 and a home-built setup) was used to acquire extinction spectra. Scanning electron microscopy (SEM) provided morphological information of the gold nanodisk arrays.

Hybrid device characterization: The plasmonic and pyroelectric responses of the devices were studied using a solar simulator (LCS-100, Oriel Instruments, $300 \mathrm{~W}$ Xe lamp, AM1.5G filter) calibrated to an irradiance of 1 Sun $\left(1000 \mathrm{~W} \mathrm{~m}^{-2}\right)$. A Keithley 4200-SCS parameter analyzer provided with two high-resolution SMUs was used to measure the short-circuit current, with the time intervals between bright/dark states set to 10 minutes. Voltage and power outputs on an external load (9 M $\Omega$ resistor) were measured using a Keithley 2182A nano-voltmeter. A custom labview program was used to collect the data. The sampling time was $1.8 \mathrm{~s}$ and $2.7 \mathrm{~s}$ for short-circuit current and output voltage measurements, respectively.

\section{Supporting Information}

Supporting Information is available from the Wiley Online Library or from the author.

\section{Acknowledgements}

The authors acknowledge financial support from the Wenner-Gren Foundations, the Swedish Research Council (2015-05070), the Swedish Foundation for Strategic Research, the ÅForsk Foundation, the Royal Swedish Academy of Sciences, and the Swedish Government Strategic Research Area in Materials Science on Functional Materials at Linköping University (Faculty Grant SFO-Mat-LiU No 2009 00971). 


\section{WILEY-VCH}

Received: ((will be filled in by the editorial staff))

Revised: ((will be filled in by the editorial staff)) Published online: ((will be filled in by the editorial staff))

\section{References}

[1] a) C. Fankhauser, J. Chory, Annu. Rev. Cell Dev. Biol. 1997, 13, 203; b) A. Cloot, Math Comput Model. 1994, 19, 23; c) D. Serafini, F. Brilli, P. Pinelli, S. Delfine, F. Loreto, J. Plant Interact. 2007, 2, 253.

[2] a) C. R. Bowen, J. Taylor, E. LeBoulbar, D. Zabek, A. Chauhan, R. Vaish, Energy Environ Sci. 2014, 7, 3836; b) A. Cuadras, M. Gasulla, V. Ferrari, Sens Actuators A Phys. 2010, 158, 132; c) D. Guyomar, G. Sebald, E. Lefeuvre, A. Khodayari, J. Intell. Mater. Syst. Struct. 2009, 20, 265; d) S. B. Lang, S. Muensit, Appl. Phys. A. 2006, 85, 125.

[3] J.-H. Lee, J. Kim, T. Y. Kim, M. S. Al Hossain, S.-W. Kim, J. H. Kim, J. Mater. Chem. A. 2016, 4, 7983.

[4] a) S. B. Lang, H. Athenstaedt, Science. 1977, 196, 985; b) S. B. Lang, Ferroelectrics. 1981, 34, 3; c) S. A. M. Tofail, Ferroelectrics. 2014, 472, 11.

[5] S. B. Lang, Ferroelectrics. 1984, 60, 311.

[6] X. Li, S.-G. Lu, X.-Z. Chen, H. Gu, X.-s. Qian, Q. M. Zhang, J. Mater. Chem. C. 2013, 1, 23.

[7] T. Park, J. Na, B. Kim, Y. Kim, H. Shin, E. Kim, ACS Nano. 2015, 9, 11830.

[8] a) P.-H. Ducrot, I. Dufour, C. Ayela, Sci Rep. 2016, 6, 19426; b) A. Kloczkowski, T. Z. Sen, in Physical Properties of Polymers Handbook, (Ed: J. E. Mark), Springer New York, New York, NY 2007, p. 787; c) D. Mao, B. E. Gnade, M. A. Quevedo-Lopez, in Ferroelectrics - Physical Effects, (Ed: M. Lallart), InTech, Rijeka 2011, p. Ch. 04.

[9] J. Kim, J.-H. Lee, J. Lee, Y. Yamauchi, C. H. Choi, J. H. Kim, $A P L$ Materials. 2017, 5, 073804.

[10] J. Li, M. Yang, X. Sun, X. Yang, J. Xue, C. Zhu, H. Liu, Y. Xia, Angew. Chem. Int. Ed. 2016, 55, 13828.

[11] G. Baffou, R. Quidant, Laser Photon. Rev. 2013, 7, 171.

[12] O. Neumann, C. Feronti, A. D. Neumann, A. Dong, K. Schell, B. Lu, E. Kim, M. Quinn, S. Thompson, N. Grady, P. Nordlander, M. Oden, N. J. Halas, Proc. Natl. Acad. Sci. U.S.A. 2013, 110, 11677.

[13] Y. Li, F. Nicoli, C. Chen, L. Lagae, G. Groeseneken, T. Stakenborg, H. W. Zandbergen, C. Dekker, P. Van Dorpe, M. P. Jonsson, Nano Lett. 2015, 15, 776.

[14] M. Gao, P. K. N. Connor, G. W. Ho, Energy Environ Sci. 2016, 9, 3151.

[15] Y. Pan, G. Tagliabue, H. Eghlidi, C. Höller, S. Dröscher, G. Hong, D. Poulikakos. 2016, 6, 37564 .

[16] H. Fredriksson, Y. Alaverdyan, A. Dmitriev, C. Langhammer, D. S. Sutherland, M. Zäch, B. Kasemo, Adv. Mater. 2007, 19, 4297.

[17] Y. Nishijima, L. Rosa, S. Juodkazis, Opt. Express. 2012, 20, 11466.

[18] Y. Xu, in Ferroelectric Materials and their Applications, Elsevier, Amsterdam 1991, p. 329.

[19] a) C.-C. Hsiao, A.-S. Siao, Sensors. 2013, 13, 12113; b) G. Vizdrik, S. Ducharme, V. M. Fridkin, S. G. Yudin, Phys. Rev. B. 2003, 68, 094113.

[20] S. Palto, L. Blinov, E. Dubovik, V. Fridkin, N. Petukhova, A. Sorokin, K. Verkhovskaya, S. Yudin, A. Zlatkin, EPL (Europhysics Letters). 1996, 34, 465.

[21] Y. Yang, W. Guo, K. C. Pradel, G. Zhu, Y. Zhou, Y. Zhang, Y. Hu, L. Lin, Z. L. Wang, Nano Lett. 2012, 12, 2833. 


\section{WILEY-VCH}

(a)
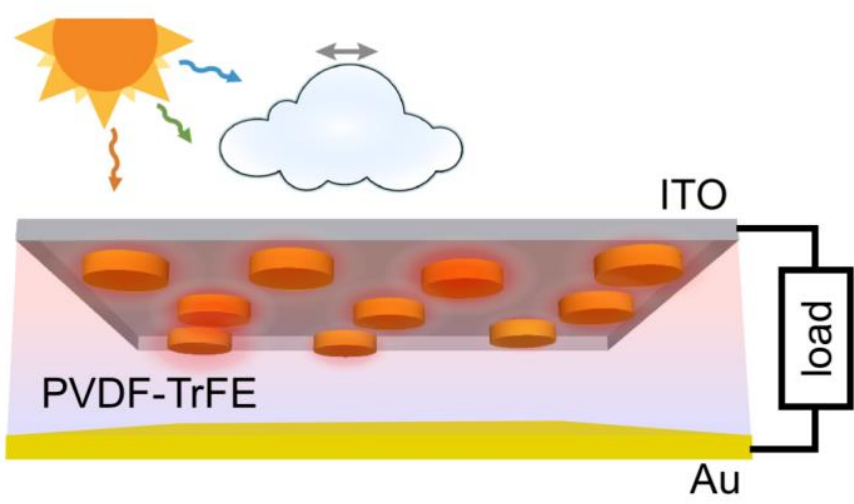

(b)
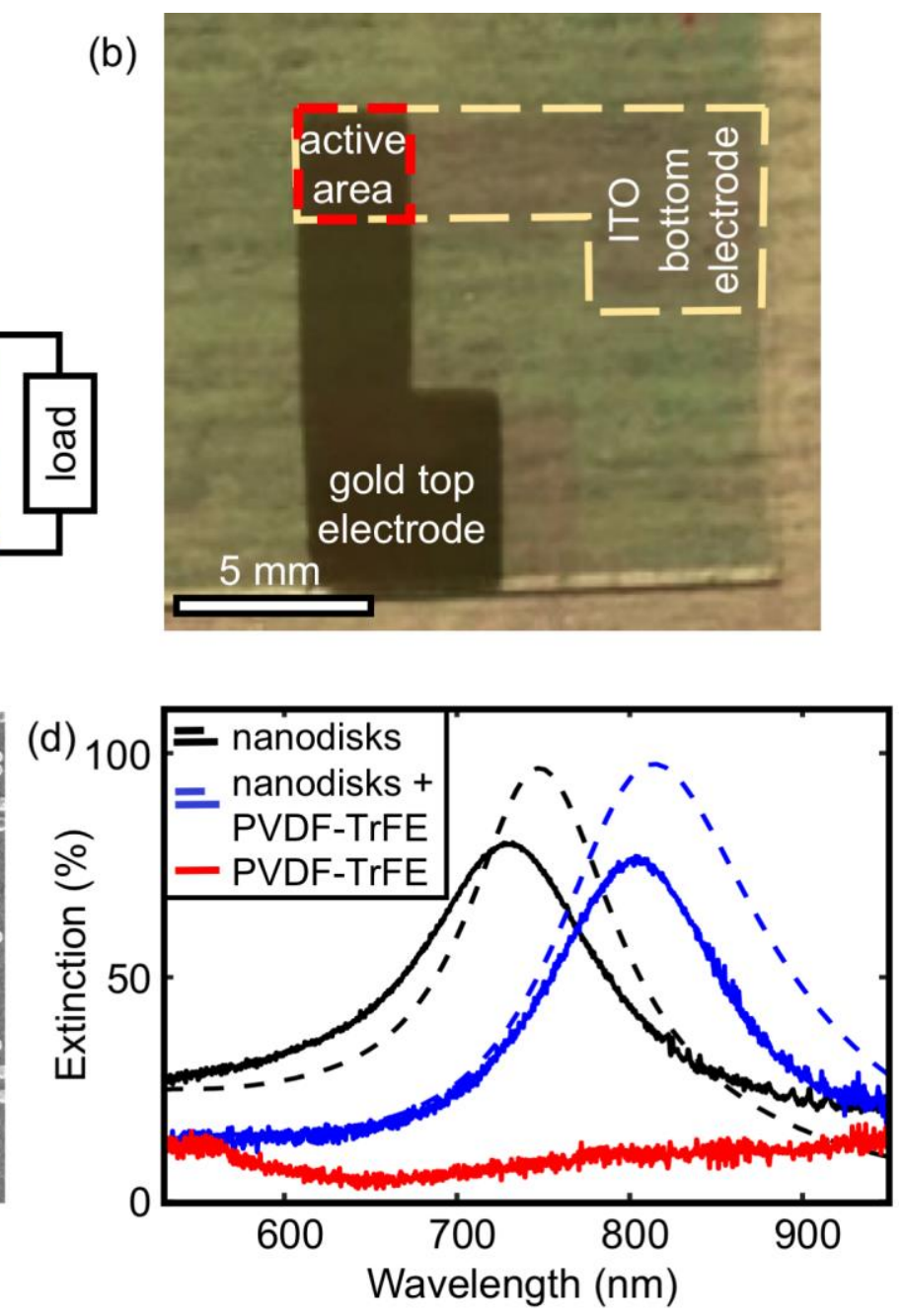

(c)

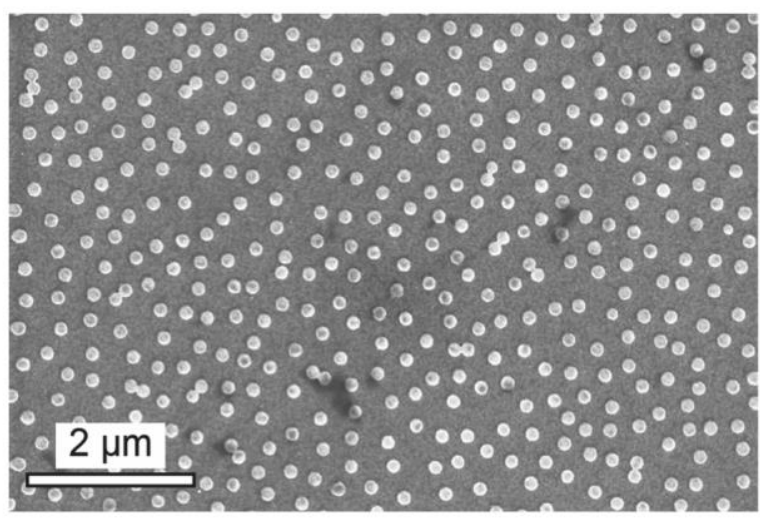

Figure 1. (a) Schematic illustration of the hybrid device (upside down and substrate omitted). (b) Photograph of a device showing the active area (red dashed square) and the bottom (yellow dashed line) and top electrodes. (c) SEM image of a typical gold nanodisk array. (d) Experimental (full lines) and simulated (dashed lines) extinction spectra of gold nanodisk arrays on ITO-covered glass, with (blue line) and without (black line) P(VDF-TrFE) copolymer. The red line shows the extinction spectra of only a P(VDF-TrFE) film. 

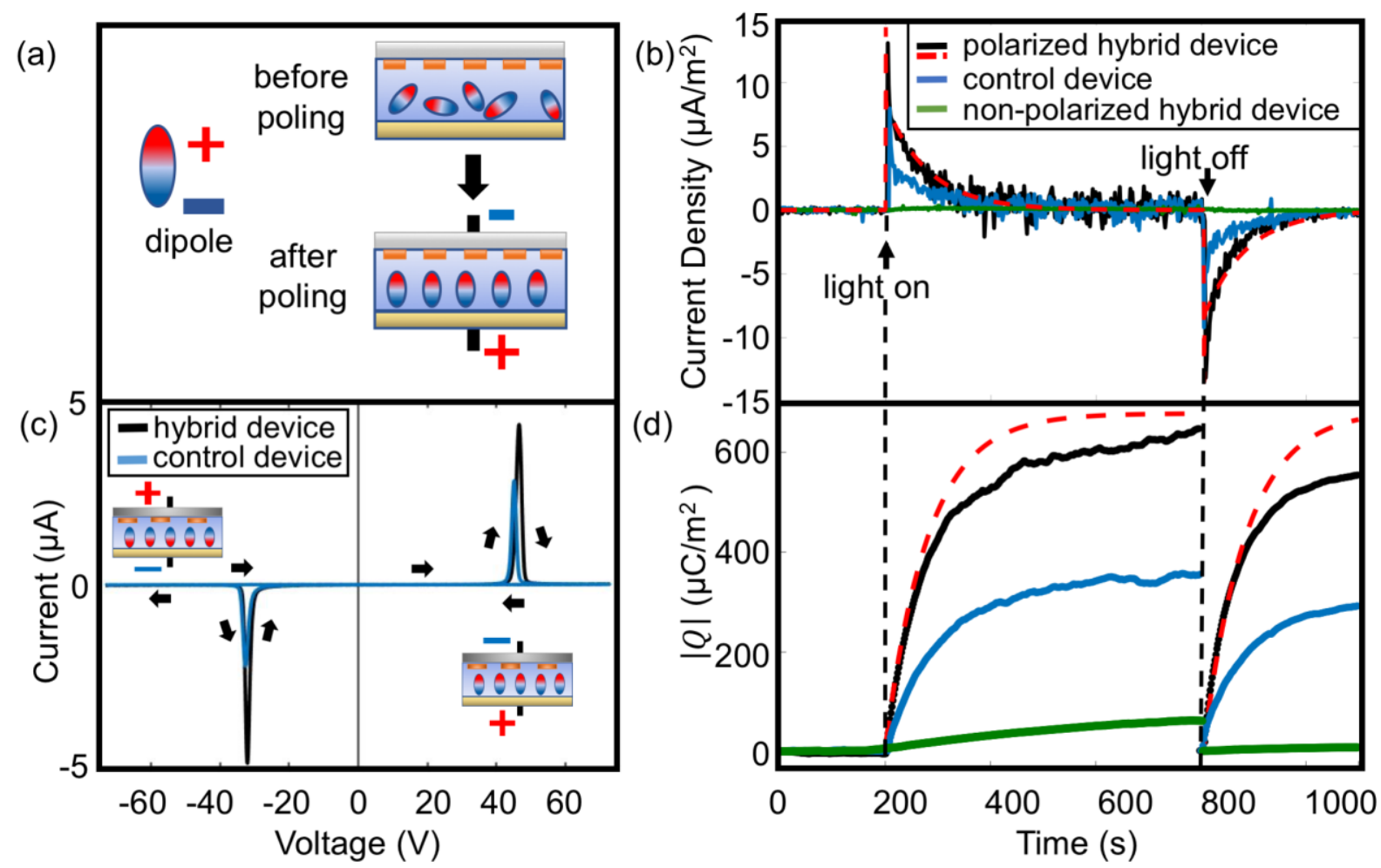

Figure 2. (a) Schematic representation of the poling process, by which the P(VDF-TrFE) domains become aligned. (b) Short-circuit current density upon fluctuating simulated solar illumination (1 Sun, on/off) for a polarized hybrid device (black line), a control device (blue line) and a non-polarized hybrid device (green line). The simulated response for the hybrid device is presented with a dashed red line. The P(VDF-TrFE) thickness was $660 \mathrm{~nm}$ for all systems. (c) Cycling voltammetry showing repolarization current as a function of bias voltage. The black line is for a hybrid device, while the blue line is for a control device without plasmonic nanostructures. (d) Alteration of absolute value of the accumulated charge density upon changes in irradiation at electrodes on a hybrid device (black line), a control device (blue line) and a non-polarized hybrid device (green line) with 660-nm thick $\mathrm{P}(\mathrm{VDF}-\mathrm{TrFE})$. The red dashed line shows the simulation result for the hybrid device. 

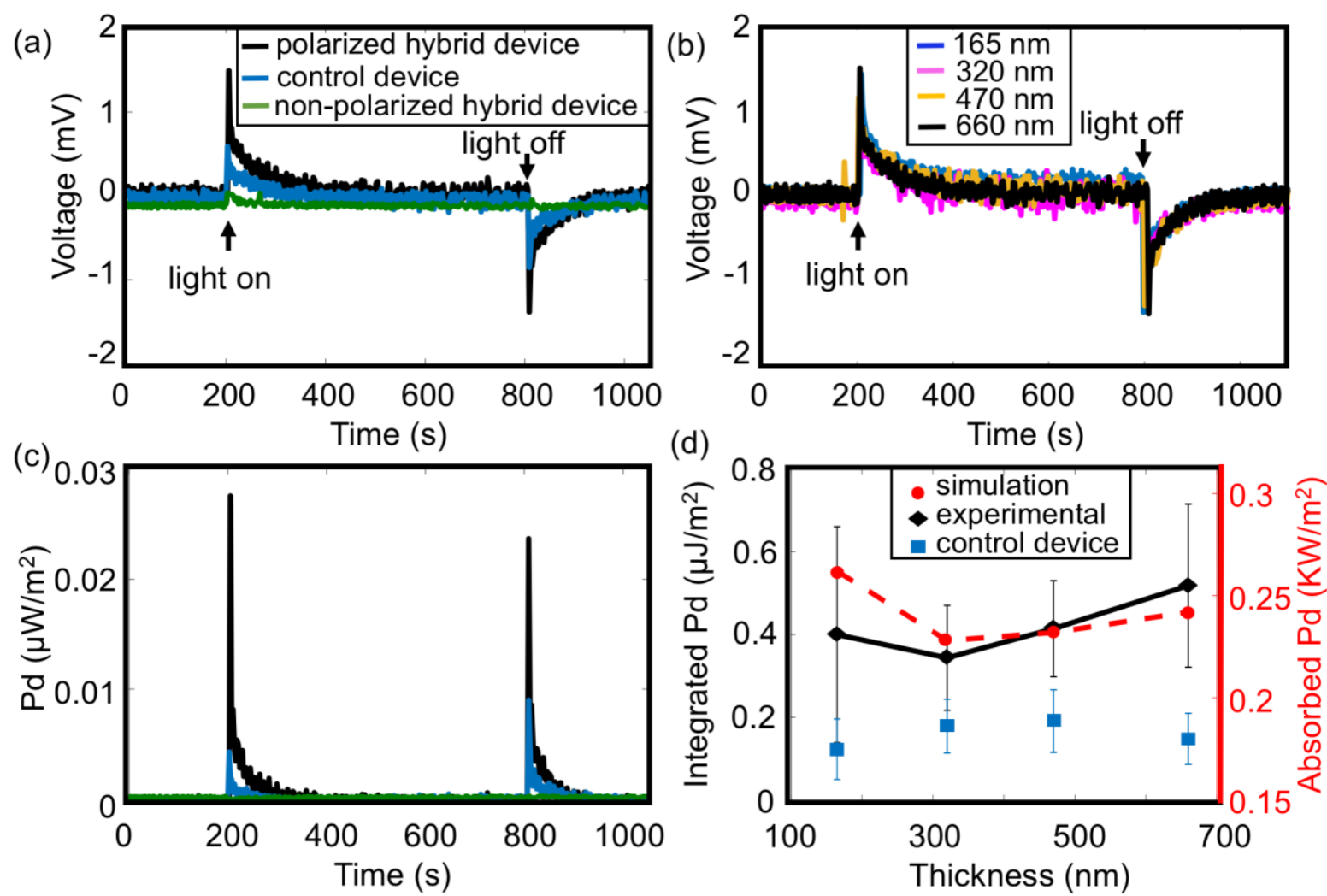

Figure 3. (a) Effect of plasmonic heating and polarization of the P(VDF-TrFE) layer on the output voltage of a polarized hybrid device (black line), a control device (blue line), and a non-polarized hybrid device (green line). The P(VDF-TrFE) thickness for all devices was $660 \mathrm{~nm}$. (b) The output voltage of hybrid devices with different P(VDF-TrFE) thicknesses (marked in the legend). The output voltages of the devices illustrated in (a) and (b) were extracted from an electrical circuit consisting the device connecting to a $9 \mathrm{M} \Omega$ load. (c) Power density of a polarized hybrid device (black line), a control device (blue line), and a non-polarized hybrid device (green line). (d) Integrated power density of hybrid devices

(black dots and line) and control devices (blue dots) with different P(VDF-TrFE) thicknesses under light on state, and simulated power density (red dots and dashed line) absorbed by each hybrid device under illumination. Each data point in experimental graph is based on at least three measurements, and symmetric error bars represent standard error. 
(a)
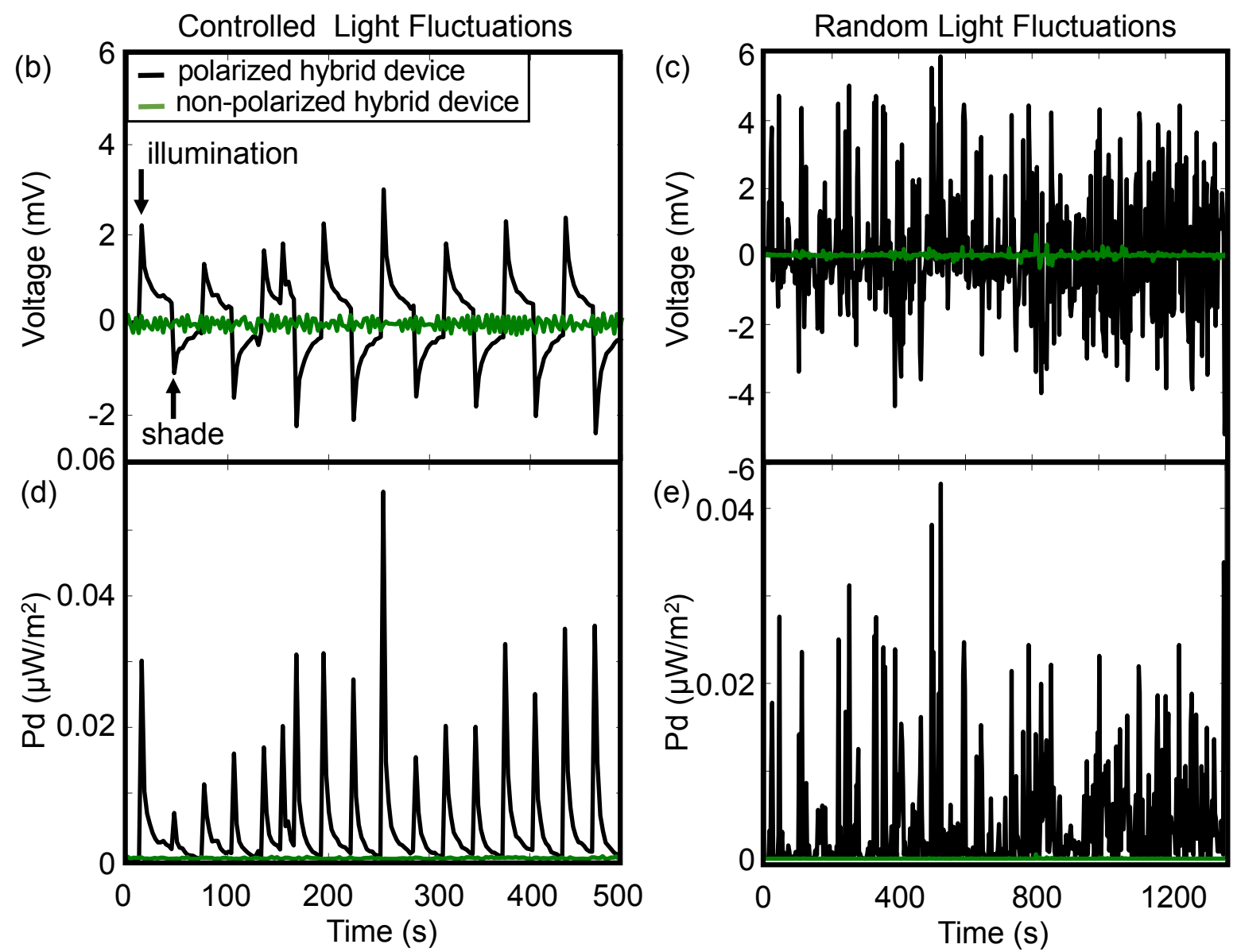

Figure 4. (a) Schematic illustration of experimental setup for random light fluctuation (1 Sun, illumination/shade) upon the hybrid device. (b) Effect of polarization of the P(VDF-TrFE) layer on the output voltage of a polarized hybrid device (black line) and a non-polarized hybrid device (green line) upon controlled light fluctuations using a leaf. (c) Output voltage of a polarized hybrid device (black

line) and a non-polarized hybrid device (green line) upon random light fluctuations using a leaf swinging in the air turbulence caused by a fan. The output voltages of the devices illustrated in (b) and (c) were extracted from an electrical circuit consisting the device connecting to a $9 \mathrm{M} \Omega$ load. (d) The power density produced by polarized hybrid device (black line) and a non-polarized hybrid device

(green line) upon controlled light fluctuations using a leaf. (e) The power density produced by 


\section{WILEY-VCH}

polarized hybrid device (black line) and a non-polarized hybrid device (green line) upon random light

fluctuations using a leaf swinging in the air turbulence caused by a fan. The thickness of the P(VDFTrFE) for all devices is $660 \mathrm{~nm}$. 


\section{WILEY-VCH}

Graphical Table of Contents

A hybrid plasmonic pyroelectric device is presented to harvest energy from temporal light A hybrid plasmonic pyroelectric device is presented to harvest energy from temporal light fluctuations. Plasmonic gold nanodisks act as light-triggered nanoscale heat sources and convert the optical energy fluctuations to thermal energy fluctuations. This induces temperature changes in a copolymer pyroelectric layer $(\mathrm{P}(\mathrm{VDF}-\mathrm{TrFE}))$, resulting in electrical output and energy harvesting of the light intensity fluctuations.

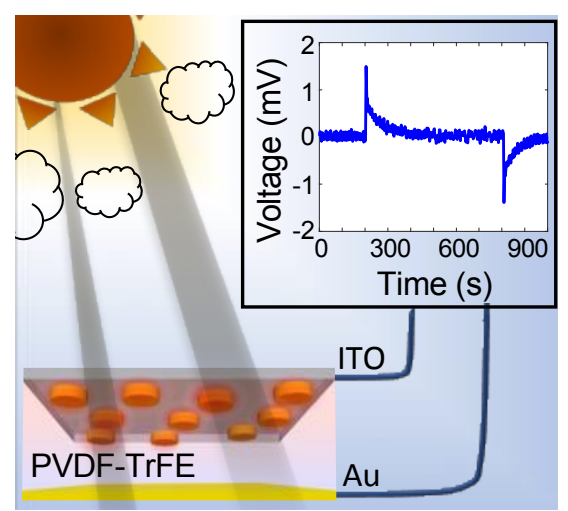




\section{WILEY-VCH}

Copyright WILEY-VCH Verlag GmbH \& Co. KGaA, 69469 Weinheim, Germany, 2016.

\section{Supporting Information}

\section{Hybrid Plasmonic and Pyroelectric Harvesting of Light Fluctuations}

Mina Shiran Chaharsoughi, Daniel Tordera, Andrea Grimoldi, Isak Engquist, Magnus Berggren, Simone Fabiano, Magnus P. Jonsson

Characteristic spacing between gold nanodisks: the spatial distribution of nanodisks on the substrates was calculated using the radial distribution function using a custom-made Matlab script and the radial distribution function (RDF) of the software ImageJ. Figure S1c shows the calculated distribution of gold nanodisks for two different SEM images (shown in Figure S1a and b) taken from different parts of a sample with gold nanodisks. There is a clear peak in the RDF at around $325 \mathrm{~nm}$, which corresponds to the typical characteristic spacing between nanodisks. The results are very similar for the two images, indicating homogeneous distribution over the substrate.

(a)

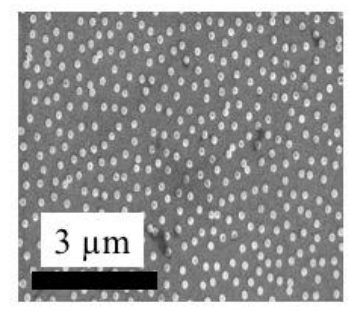

(b)

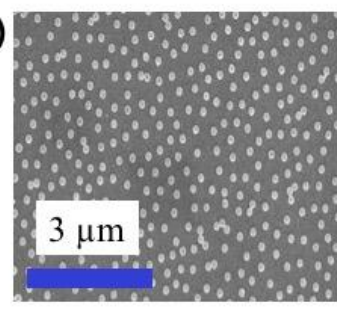

(c)

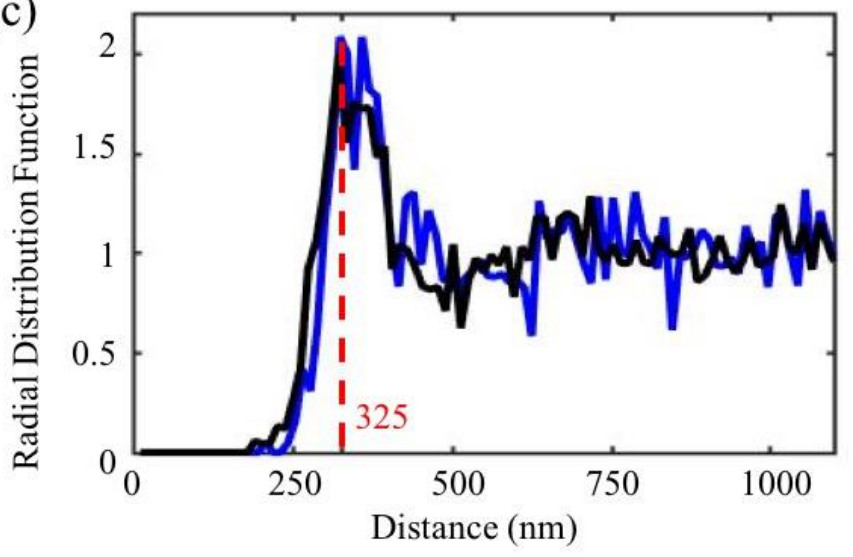

Figure S1. (a) and (b) SEM images of gold nanodisks obtained from two different regions of a typical sample. (c) Radial distribution function of the gold nanodisks, with the black and blue lines corresponding to (a) and (b), respectively. The peak at around $325 \mathrm{~nm}$ corresponds to the characteristic nearest neighbor spacing between nanodisks. 


\section{WILEY-VCH}

Optical and Heat Transfer Simulations: we carried out optical simulations of the different systems using the finite-difference time-domain (FDTD) methodology (FDTD Solutions, Lumerical). The geometry of the system was as follows: a glass substrate (refractive index of 1.5), a $90 \mathrm{~nm}$ thick ITO layer (averaged refractive index of 1.65), a $158 \mathrm{~nm}$ diameter and $30 \mathrm{~nm}$ thick nanodisk (refractive index given by the software's database, Johnson and Christy). For complete hybrid devices, we added a P(VDF-TrFE) layer (refractive index of 1.42, thickness of $165 \mathrm{~nm}, 320 \mathrm{~nm}, 470 \mathrm{~nm}$ or $660 \mathrm{~nm}$,) and a top gold film with a thickness of $30 \mathrm{~nm}$. The unit cell was $325 \times 325 \mathrm{~nm}^{-2}$ with periodic boundary conditions in both $x$ and $y$ directions, and perfectly matched layers in the $z$ direction (normal to the substrate). We used a plane-wave propagating along the $z$ axis as a light source, with a wavelength range of 400-1000 nm. The response of each system was analyzed by transmission and reflection monitors as well as using advanced analysis groups to obtain the absorbed power in the gold nanodisks and the gold film. Figure S2a to d, show the simulated (dashed lines) and experimental (solid lines) extinction spectra of hybrid devices with different P(VDF-TrFE) thicknesses $(165 \mathrm{~nm}, 320 \mathrm{~nm}, 470 \mathrm{~nm}$ or $660 \mathrm{~nm}$ ). The results show that the simulations and experiments graphs are in good agreement.
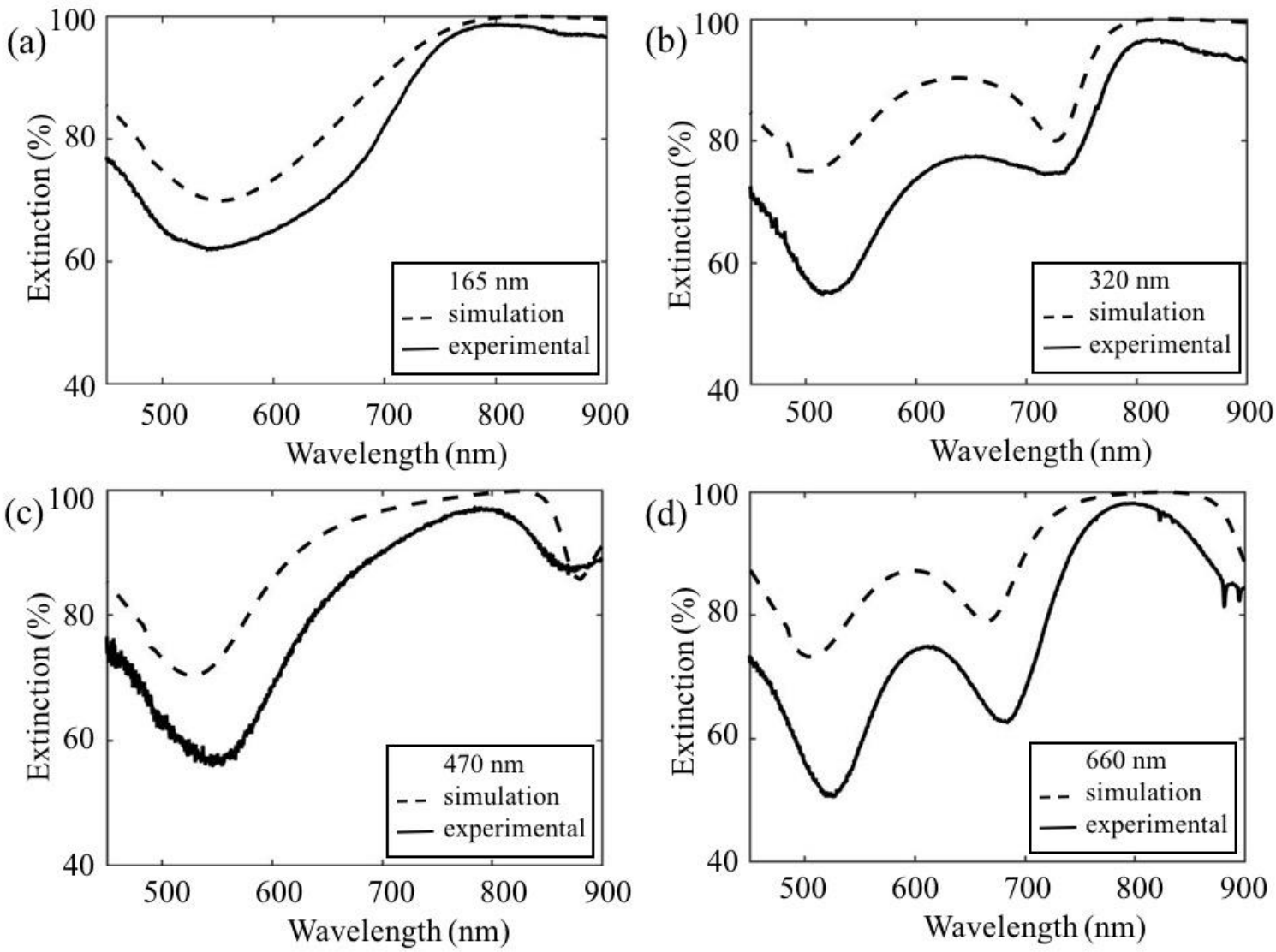

Figure S2. Simulated (dashed line) and experimental (full line) extinction spectra of hybrid devices with P(VDF-TrFE) thicknesses of (a) $165 \mathrm{~nm}$, (b) $320 \mathrm{~nm}$, (c) $470 \mathrm{~nm}$ and (d) $660 \mathrm{~nm}$.

The temperature variations over time were studied using the finite element method (FEM), using COMSOL Multiphysics (version 5.1). The same geometry as aforementioned was used, albeit including the full thickness of the glass substrate of $1 \mathrm{~mm}$. We incorporated the absorbed powers obtained in the optical simulations as heat sources in the gold nanodisk and film. The simulations used periodic boundary conditions in $x$ and $y$ directions, and a convective heat flux 


\section{WILEY-VCH}

in $z$, with a heat transfer coefficient of $10 \mathrm{~W} \mathrm{~m}^{-2} \mathrm{~K}^{-1} \cdot{ }^{[1]}$ The thermal conductivity, heat capacity and density of gold and glass were provided by the software material database. The thermal conductivity, heat capacity and density of ITO and P(VDF-TrFE) were obtained from literature values. ${ }^{[2]}$ Time-dependent simulations were carried out with explicit events to switch on/off the heat (i.e. light) source. The temperature was evaluated under the gold nanodisks. Figure S3 illustrates the simulation results for a hybrid device with a $660 \mathrm{~nm}$ P(VDF-TrFE) thickness.
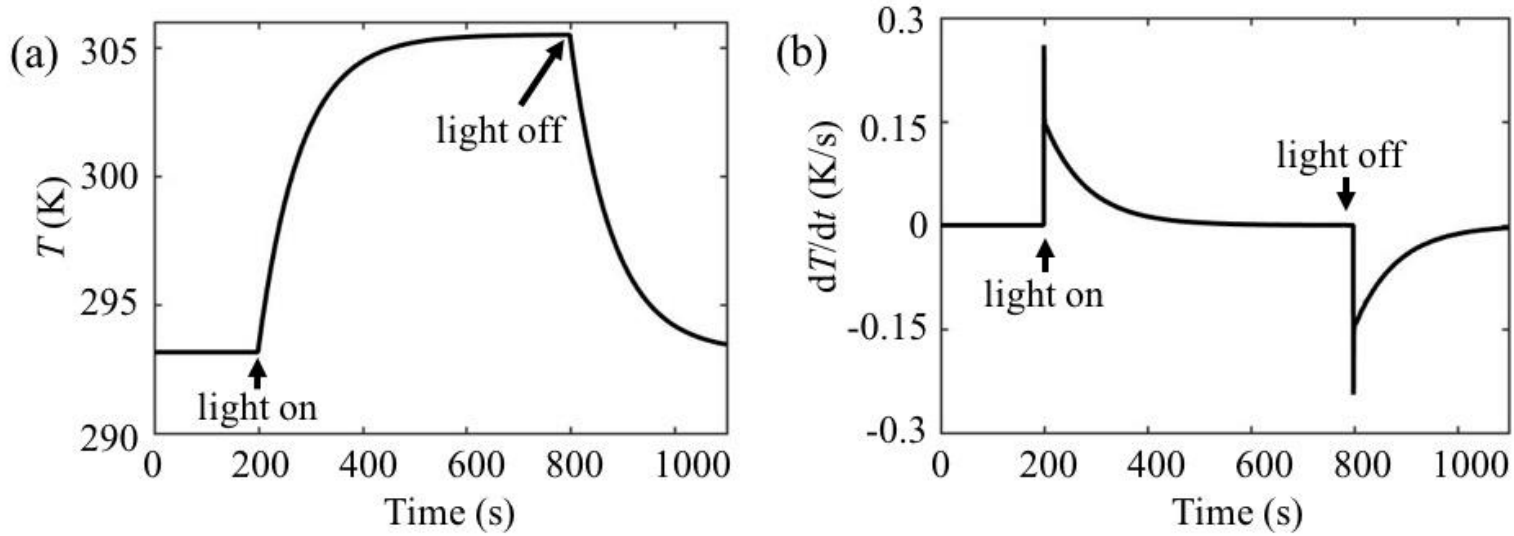

Figure S3. (a) Time-dependent simulated temperature evolution for a hybrid device $(660 \mathrm{~nm}$ $\mathrm{P}(\mathrm{VDF}-\mathrm{TrFE})$ thickness) upon intermittent solar illumination. (b) Calculated rate of change in temperature extracted from (a).

[1] E. s. Edge, Convective Heat Transfer Coefficient Table Chart, http://www.engineersedge.com/heat_transfer/convective_heat_transfer_coefficients 13378.htm., accessed: Month, 2017.

[2] a) M. Abb, P. Albella, J. Aizpurua, O. L. Muskens, Nano Lett. 2011, 11, 2457; b) T. Ashida, A. Miyamura, N. Oka, Y. Sato, T. Yagi, N. Taketoshi, T. Baba, Y. Shigesato, J. Appl. Phys. 2009, 105, 073709; c) J. A. Chilton, in Plastics for Electronics, (Ed: M. Goosey), Springer Netherlands, Dordrecht 1999, p. 243; d) N. Matsunaga, S. Ueno, Y. Tanaka, Y. Hirata, in Characterization and Control of Interfaces for High Quality Advanced Materials II, John Wiley \& Sons, Inc., 2006, p. 153; e) V. K. Varadan, K. J. Vinoy, S. Gopalakrishnan, in Smart Material Systems and MEMS, John Wiley \& Sons, Ltd, 2006, p. 43. 